\title{
Stärkung sozialer Rechte durch Grundrechtsschutz im europäischen Mehr-Ebenen-System?
}

\author{
Von Monika Schlachter, IAAEU, Trier*
}

Ob soziale Rechte überhaupt als justitiable Grundrechte gewährleistet werden, ist vielfach umstritten; auch die Europäische Grundrechtecharta hat sich dazu nicht eindeutig positioniert. Auf völkerrechtlicher Ebene existieren allerdings zahlreiche Instrumente, die soziale Rechte garantieren. Zu denjenigen Übereinkommen, die von den EU-Mitgliedstaaten am häufigsten akzeptiert worden sind, gehören neben der Europäischen Sozialcharta und der Europäischen Menschenrechtskonvention zahlreiche Übereinkommen der Internationalen Arbeitsorganisation. Aus der Vervielfachung von Rechten folgt ein Koordinierungsproblem, denn eine Abstimmung verschiedener parallel geltender Gewährleistungen gelingt trotz bestehender Kooperationsklauseln keineswegs stets spannungsfrei. Das gilt insbesondere bei Beteiligung der Unionsebene als supranationale Rechtsordnung mit entsprechenden Besonderheiten: Die EU unterliegt zwar bei der Garantie von sozialen Rechten deutlichen Begrenzungen ihrer Kompetenz, um die Sozialpolitik der Mitgliedstaaten gegen Eingriffe des Unionsrechts abzusichern. Bei der Einschränkung sozialer Rechte, die auf mitgliedstaatlicher oder auf völkerrechtlicher Ebene gewährleistet werden, gilt aber der Vorrang des Unionsrechts gegenüber allem einzelstaatlichen Recht. Auch wo die EU selbst nicht effektiv schützen oder gewährleisten kann, kann sie effektiv beschränken. Durch diesen Mechanismus können soziale Rechte erheblich geschwächt, und damit die Mitgliedstaaten in Konflikt mit bestehenden völkerrechtlichen Verpflichtungen gebracht werden. Um unerwünschte Konsequenzen zu verringern oder zu vermeiden, bedarf es einer besseren Abstimmung auf allen beteiligten Ebenen, um eine international stärker angenäherte Auslegung sozialer Rechte zu erreichen.

\section{Einführung}

Die Gewährleistung von Grundrechten, einschließlich sozialer Rechte, ist längst nicht mehr allein den nationalen Verfassungen vorbehalten. In den Mitgliedstaaten der EU ist die Europäische Grundrechtecharta (GRC) hinzugetreten und für die Mitglieder des Europarates gelten zusätzlich die Europäische Menschenrechtskonvention ${ }^{1}$ (EMRK) und die Europäische Sozialcharta ${ }^{2}$ (ESC). Aus dieser

\footnotetext{
* Prof. Dr. Dr. h.c. Monika Schlachter ist Direktorin des IAAEU und Professorin des Fachbereichs Rechtswissenschaft an der Universität Trier; der Beitrag beruht auf einem Vortrag auf der $1^{\text {st }}$ Conference on International Labour Law, 25. November 2015, veranstaltet von Hugo-Sinzheimer-Institut - HSI - (Frankfurt) und Institut für Arbeitsrecht und Arbeitsbeziehungen in der Europäischen Union - IAAEU - (Trier).

1 Europäische Menschenrechtskonvention, in Kraft getreten am 4.11.1950.

2 Europäische Sozialcharta vom 18.10.1961, in Kraft getreten am 26.2.1965; revidierte Europäische Sozialcharta vom 3.5.1996, in Kraft getreten am 1.7.1999.
} 
mehrstimmigen Grundrechtsgewährleistung erwächst ein Vorrangs- und Abstimmungsproblem. In allen Anwendungsfällen, für die es an verbindlichen Kollisionsregeln fehlt, sind die zur Kontrolle berufenen Gerichte um ein Kooperationsverhältnis bei der Auslegung und Anwendung von Grundrechtsgarantien bemüht. Ziel dieser Kooperation ist die Sicherung eines hohen Schutzniveaus für die Bürger, aber auch die Verhinderung von Pflichtenkollisionen für Staaten, die parallel an mehrere Gewährleistungssysteme gebunden sind. Dabei soll der bereits erreichte Standard gesichert statt in Folge der Etablierung eines zusätzlichen Schutzinstruments gesenkt werden. ${ }^{3}$

Wie sich eine Auslegungskooperation der Gerichte praktisch vollzieht, wird sowohl von deren Selbstverständnis als auch von der relativen Durchsetzungsfähigkeit der Grundrechtsgewährleistungen mitbestimmt. Unter Umständen ignoriert ein Gericht bei der Interpretation von Grundrechten im eigenen Zuständigkeitsbereich das Vorhandensein von parallelen Gewährleistungen in internationalen Verträgen vollständig oder begnügt sich mit einem Hinweis auf ihre Existenz, ohne sich inhaltlich damit auseinanderzusetzen. Im kooperationsfreundlichsten Fall wird bei der Auslegung dagegen differenziert geprüft, welche internationalen Quellen vergleichbare Rechte gewährleisten, welcher Gehalt ihnen zugemessen wird und unter welchen Voraussetzungen sie eingeschränkt werden können. Daran schließt sich die Überlegung an, ob die Umstände der Grundrechtsgewährleistung in den beteiligten Rechtsinstrumenten ähnlich genug sind, um einen gemeinsamen Interpretationsstandard zu begründen, oder ob eine gewichtige Besonderheit besteht, die eine abweichende Auslegung geboten erscheinen lässt. Auch beim Umfang der Grundrechtsgewährleistung geht es typischerweise um die Garantie von Mindestschutz: Sofern jedenfalls das Schutzniveau gewährleistet bleibt, das im Zuständigkeitsbereich des kontrollierenden Gerichts garantiert ist, bestehen keine Vorbehalte gegen die Verstärkung des Grundrechtsschutzes durch andere völkerrechtliche Verträge. ${ }^{4}$

Dieser Ansatz zur Konfliktvermeidung ist allerdings für Kollisionslagen unter Beteiligung der EU nur teilweise geeignet. ${ }^{5}$ Soweit das Unionsrecht den Mitgliedstaaten einen Regelungsspielraum belässt, den sie nach Maßgabe ihrer völkerrechtlichen Bindungen ausfüllen können, steht es in ihrer Macht, einen Konflikt etwa mit den sozialen Rechten der ESC zu vermeiden. ${ }^{6}$ Wenn Mitgliedstaaten trotz bestehendem Entscheidungsspielraum eine Minimallösung wählen, die zwar vom EU-Recht noch gedeckt ist, aber ihren internationalen Pflichten widerspricht,

3 T. v. Danwitz, in: Tettinger/Stern (Hrsg.), Kölner Gemeinschaftskommentar zur EU-Grundrechtecharta, 2006, Art. 53 GRC, Rn. 8 f.; M. Borowski, in: Meyer (Hrsg.), Charta der Grundrechte der EU, 4. Aufl. 2014, Art. 53 GRC, Rn. 7; J. Terhechte, in: v. d. Groeben/Schwarze/Hatje (Hrsg.), Europäisches Unionsrecht, 7. Aufl. 2015, Art. 53 GRC, Rn. 1.

4 EGMR, Entsch. v. 5.10.1999 - 44958/98 (Anderson/UK), Rn. 31; Entsch. v. 29.11.2001 - 42246/98 (Johnson/ UK), Rn. 1.

5 T. Kingreen, in: Calliess/Ruffert (Hrsg.), EUV/AEUV, 2011, Art. 53 GRC, Rn. 5; K. Lenaerts, Kooperation und Spannung im Verhältnis von EuGH und nationalen Verfassungsgerichten, EuR 2015, S. 3, 24.

6 EuGH, Urteil v. 27.6.2006, Rs. C-540/03 (Parlament/Rat), ECLI:EU:C:2006:429, Slg. 2006, I-05769, Rn. 107. 
ist dies eine politische Entscheidung auf nationaler Ebene, die von der Mitgliedschaft in der Union nicht bedingt wird. ${ }^{7}$ Soweit die EU jedoch die Rechtsordnungen nicht nur koordiniert, sondern harmonisiert, regeln diese Vorgaben keine Mindestgewährleistungen. Vielmehr setzt eine Vollharmonisierung Standards, von denen im Recht der Mitgliedstaaten nicht abgewichen werden darf. ${ }^{8}$ Besonders relevant wird dies für solche kollidierenden Grundrechte oder Sekundärrechtsakte, die soziale Rechte begrenzen. ${ }^{9}$ Nachfolgend sollen Wege dargelegt werden, solche Kollisionsfälle zu verringern.

\section{Lösungen im Unionsrecht}

\section{Querschnittsklauseln der GRC}

Die Union hat in der Grundrechtecharta selbst Bestimmungen zur Auflösung von Kollisionslagen zwischen verschiedenen Ebenen der Grundrechtsgewährleistung geschaffen, vorrangig die Querschnittsbestimmungen in Art. 52 Abs. 3 und 53 GRC. Im Verhältnis zur Menschenrechtskonvention bedient sich Art. 52 Abs. 3 GRC dabei des Mittels, die Konventionsbestimmungen zugleich als Mindestinhalt damit korrespondierender Rechte in die GRC zu übernehmen. Damit kann eine Unterschreitung des Schutzniveaus der EMRK vermieden werden und zugleich die Eigenständigkeit des Unionsrechts im darüberhinausgehenden Bereich gewährleistet werden. Der Unterschied zur „Regelungstechnik“ der EMRK ist jedoch signifikant: Statt anderen Grundrechtsinstrumenten die Möglichkeit zur Gewährung weitergehenden Schutzes zu belassen, behält die GRC es der Union vor, ihrerseits das Schutzniveau der EMRK zu überschreiten. Zu einer unmittelbaren Kollisionslage führt dies zunächst nicht, da die EMRK es den Konventionsstaaten freistellt, ein höheres Schutzniveau beizubehalten oder einzuführen, Art. 53 EMRK. Ein von der GRC weitergehend gewährleisteter und von den Mitgliedstaaten umgesetzter Grundrechtsschutz würde vom EGMR also nicht beanstandet, während sich die Konventionsstaaten zwar dem EGMR, nicht aber dem EuGH gegenüber darauf berufen dürften, selbst die EMRK übersteigende Schutzstandards eingeführt zu haben; das schafft jedenfalls mittelbaren Anlass für Konflikte, da sich die Konventionsstaaten in Widerspruch zum Vorrang des Unionsrechts setzen könnten.

Im Verhältnis der GRC zu anderen völkerrechtlichen Verträgen, denen kein vergleichbarer Status wie der EMRK eingeräumt wird, sind die Bedingungen für die Auflösung von Kollisionslagen komplexer. Art. 52 Abs. 3 GRC greift hier nach

7 EuGH, Urteil v. 27.6.2006, Rs. C-540/03 (Fn. 6), Rn. 107; vgl. weiter: H. Jarass, Charta der Grundrechte der EU, 2. Aufl. 2013, Art. 53 GRC, Rn. 10.

8 C. Ladenburger, in: Tettinger/Stern (Fn. 3), Art. 53 GRC, Rn. 31; J. Terhechte, in: v. d. Groeben/Schwarze/ Hatje (Fn. 3), Art. 53 GRC, Rn. 3.

9 EuGH, Urteil v. 15.7.2010, Rs. C-271/08 (KOM/Deutschland), ECLI:EU:C:2010:426, Slg. 2010, I-07091, Rn. 66. 
überwiegender Ansicht nicht, auch nicht in entsprechender Anwendung, da die Sonderstellung für die EMRK bewusst eingerichtet worden ist. ${ }^{10}$ Die ersatzweise bereitgestellte Regelung in Art. 53 GRC soll allerdings ebenfalls verhindern, dass durch die GRC der internationale Grundrechtsschutz in den Mitgliedstaaten beeinträchtigt werden könnte. Daher dürfen völkerrechtliche Abkommen, denen alle Mitgliedstaaten beigetreten sind ${ }^{11}$, durch eine Auslegung der GRC nicht eingeschränkt oder nachteilig betroffen werden. Zu diesen Abkommen gehört auch die $\mathrm{ESC}^{12}$, die alle Mitgliedstaaten entweder in ihrer ursprünglichen oder ihrer revidierten Fassung ratifiziert haben. ${ }^{13}$ Daraus ist jedoch nicht auf die Unanwendbarkeit von Art. 53 GRC zu schließen. Dass der Ratifizierungsstand nicht zu allen Vorschriften identisch ist, ist vornehmlich der Konstruktion der Sozialcharta geschuldet, die den Staaten eine a-la-carte-Ratifizierung gerade freistellt ${ }^{14}$; darüberhinausgehend ist eine weitgehende Einheitlichkeit erreicht: Die revidierte Fassung der ESC baut auf der älteren auf, die lediglich aktualisiert und konkretisiert wird; zu Widersprüchen oder Einschränkungen im Grundrechtsbestand führt das nicht. Dass die Anwendbarkeit von Art. 53 GRC keinen einheitlichen Ratifizierungsstand voraussetzt, zeigt sich auch mit Blick auf die EMRK, deren Zusatzprotokolle ebenfalls nicht von allen Staaten ratifiziert worden sind. ${ }^{15}$

Fällt die Sozialcharta in den Anwendungsbereich von Art. 53 GRC, darf eine Auslegung von Bestimmungen der GRC sie weder einschränken noch beeinträchtigen. Verboten wird damit aber nicht die Einschränkung im Ergebnis, sondern eine Auslegung von Bestimmungen der GRC, die solche Nachteile bewirkt. Gerade die sozialen Grundrechte, die oft auf Unionsrecht oder das Recht der Mitgliedstaaten zurückverweisen, geben entsprechend seltener selbst unmittelbar die Möglichkeit für eine einschränkende Auslegung, sondern nehmen die an anderer Stelle enthaltenen Einschränkungen in Bezug. Eine auf andere Weise durch das Unionsrecht bewirkte Einschränkung oder Verringerung des Schutzniveaus der Sozialcharta wird von Art. 53 GRC nicht ausgeschlossen. ${ }^{16}$ Insbesondere der Anspruch des Unionsrechts auf Vorrang gegenüber allem nationalen Recht fällt nicht unter dieses Verbot, denn er beruht nicht auf einer Auslegung der GRC.

Werden soziale Rechte in der Union durch Sekundärrechtsakte konkretisiert oder beschränkt, die als Harmonisierungsinstrument verstanden werden, sind grund-

10 M. Borowski, in: Meyer (Fn. 3), Art. 53 GRC, Rn. 9.

11 Auf eine Ratifizierung solcher Abkommen durch sämtliche Mitgliedstaaten soll es dabei nicht ankommen, solange diese nur am Abschluss des Übereinkommens beteiligt waren: P. Tettinger/K. Stern/T. v. Danwitz, Europäische Grundrechtecharta, Art. 53 GRC, Rn. 17; M. Borowski, in: Meyer (Fn. 3), Art. 53 GRC, Rn. 18 f.

12 EuGH, Rs. C-116/06 (Kiiski), ECLI:EU:C:2007:536, Slg. 2007, I-07643, Rn. 48.

13 Vier Mitgliedstaaten sind ausschließlich an die ESC (1961) gebunden; fünf weitere haben neben der ESC (1961) noch das Zusatzprotokoll von 1988 ratifiziert; die übrigen 19 Mitgliedstaaten sind an die revidierte ESC (1996) gebunden.

14 M. Schlachter, Could the ESC contribute to a more social Europe?, in: Countouris/Freedland (Hrsg.), Resocialising Europe in time of crisis, 2013, S. 105, $107 \mathrm{ff}$.

15 T. v. Danwitz, in: Tettinger/Stern (Fn. 3), Art. 52 GRC, Rn. 59; Art. 53 GRC, Rn. 17.

16 B. de Witte in: Peers/Hervey/Kenner/Ward, The EU Charter of Fundamental Rights, 2014, Part I, Art. 53 GRC, Rn. 53.21. 
rechtsverstärkende Abweichungen nicht zulässig: Günstigere Mindestarbeitsbedingungen als in der Entsende-Richtlinie vorgesehen darf ein Tarifvertrag den Entsendunternehmen nach der etablierten Rechtsprechung nicht vorschreiben ${ }^{17}$. Zwar hat der EuGH mittlerweile die Schaffung differenzierender tariflicher Mindestentgeltsätze ermöglicht ${ }^{18}$, dass daraus aber eine künftige Anwendung des Günstigkeitsgrundsatzes aus Art. 3 Abs. 7 UA. 1 RL 96/71/EG abgeleitet werden kann, steht keineswegs fest. ${ }^{19}$ Ein Mitgliedstaat darf den Entsendeunternehmen auch nach aktueller Rechtsprechung die Einhaltung von Tarifverträgen nicht in weiterem Umfang vorschreiben als die Richtlinie. ${ }^{20}$ Das gilt unabhängig davon, dass Art. 6 ESC eine gesetzliche Beschränkung von Tarifinhalten oder ein Verbot von Arbeitskämpfen zur Durchsetzung besserer Arbeitsbedingungen grundsätzlich nicht akzeptiert. Da Mitgliedstaaten beiden Anforderungen parallel verpflichtet sind, kann es trotz Art. 53 GRC zur Kollision zwischen einem grundrechtsbeschränkenden Sekundärrechtsakt der EU und Verpflichtungen der Mitgliedstaaten aus der ESC kommen. ${ }^{21}$

\section{Pflicht zum Beitritt zur EMRK, Art. 6 Abs. 2 EUV}

Ein anderes Instrument zur Vermeidung potentieller Grundrechtskollisionen stellt Art. 6 Abs. 2 EUV bereit, der die EU auf einen Beitritt zur Menschenrechtskonvention verpflichtet. Das lässt sich durchaus im Sinne der Erforderlichkeit eines einheitlichen Gewährleistungsniveaus interpretieren, denn die Union will sich damit ebenso wie ihre Mitgliedstaaten an die Menschenrechte binden. Wäre die Union selbst Konventionspartei, würden die Konventionsgrundrechte nicht mehr nur als Mindestgehalt der GRC durch den EuGH gesichert, sondern könnten unmittelbar vor dem EGMR geltend gemacht werden. Auch Individualbeschwerden von Bürgern gegen eine Verletzung ihrer Rechte durch Organe der Union würden möglich. Die Anwendung oder Auslegung des Unionsrechts müsste sich im Überschneidungsbereich im Rahmen der Vorgaben der Konvention bewegen. Das könnte sich auf die weitreichenden Möglichkeiten zur Beschränkung von Grundrechten durch Sekundärrecht auswirken, die dann den Anforderungen an Schranken für Konventionsrechte genügen müssten.

Weil die EMRK den Konventionsstaaten die Überschreitung des von ihr selbst gewährleisteten Schutzniveaus ermöglicht, Art. 53 EMRK, könnten die Staaten versuchen, sich auch gegenüber der Union als anderer Vertragspartei auf diese Öffnungsklausel zu berufen. Diese Konsequenz eines Beitritts hat der EuGH in

17 EuGH, Urteil v. 18.12.2007, Rs. C-341/05 (Laval), ECLI:EU:C:2007:809, Slg. 2007, I-11767, Rn. 70 f.; Urteil v. 3.4.2008, Rs. C-346/06 (Rüffert), ECLI:EU:C:2008:189, Slg. 2008, I-01989.

18 EuGH, Urteil v. 12.2.2015, Rs. C-396/13 (Sähköalojen ammatiliitto), ECLI:EU:C:2015:86, Rn. 43 f.

19 Eine Abweichung zur gegenteiligen Aussage in der Rs. C-341/05 (Laval) (Fn. 17), Rn. 80, ist nicht ausdrücklich statuiert worden.

20 EuGH, Urteil v. 17.11.2015, Rs. C-115/14 (Regio Post), ECLI:EU:C:2015:760, Rn. 71 ff.

21 T. Kingreen, in: Calliess/Ruffert (Fn. 5), Art. 53 GRC, Rn. 4; J. Terhechte, in: v. d. Groeben/Schwarze/Hatje (Fn. 3), Art. 53 GRC, Rn. 3; M. Borowski, in: Meyer (Fn. 3), Art. 53 GRC, Rn. 22. 
seinem Gutachten zum Beitrittsvertrag beanstandet, weil er die Besonderheiten des Unionsrechts damit gefährdet sieht ${ }^{22}$, die weder durch die EMRK, noch durch die Verfassungen der Mitgliedstaaten oder andere völkerrechtliche Verpflichtungen beeinträchtigt werden dürfen. ${ }^{23}$ Auch wird Art. 53 EMRK als mit den Anforderungen der Einheit und Wirksamkeit des Unionsrechts nicht vereinbar eingeordnet. ${ }^{24}$ Vor dem Hintergrund dieses, die EU nach Art. 218 Abs. 11 AEUV bindenden, Gutachtens ist mit einem baldigen Vollzug des Beitritts nicht zu rechnen. Im Verhältnis zur Sozialcharta ist eine vergleichbare Lösung zwar nicht ausgeschlossen $^{25}$, aber eben auch nicht vorgesehen. Obwohl alle EU-Mitgliedstaaten die ESC in einer ihrer Fassungen akzeptiert haben, zieht die Union einen Beitritt zur Sozialcharta weder in Betracht noch hat sie sich gar dazu verpflichtet. Die vereinheitlichende Wirkung, die für die EMRK von einem Beitritt ausgehen könnte, ist also für die ESC nicht einmal angedacht. Der Frage, ob dem denn dieselben Bedenken hinsichtlich Einheitlichkeit und Vorrang des Unionsrechts entgegenstehen müssten, die der EuGH gegen den Konventionsbeitritt erhoben hat, soll daher nicht weiter nachgegangen werden.

Überschneidungen zwischen der GRC und der Sozialcharta bestehen aber bereits unabhängig davon gem. Art. 151 Abs. 1 AEUV, der die Sozialpolitik der Union auf die ESC stützt; weiter ist der Titel „Solidarität“ der GRC in vielen Aspekten nach dem Vorbild der ESC formuliert worden. Die verbleibenden Unterschiede sind allerdings gewichtig, nicht nur bezogen auf nicht übernommene Rechte ${ }^{26}$, sondern insbesondere auf die Einschränkung ihres Gewährleistungsgehalts. Soziale Rechte gewährleistet die Union oft als „Grundsätze“ mit eingeschränkter Justitiabilität, Art. 52 Abs. 5 GRC. Werden sie, wie Art. 27, 28 und 30 GRC, nur nach Maßgabe des Unionsrechts oder nach mitgliedstaatlichem Recht oder Gepflogenheiten garantiert, ist ihre Steuerungswirkung auf die Organe der Union wie auf die Mitgliedsaaten reduziert. ${ }^{27}$ Weiter ist eine Grundrechtskontrolle in der Union davon abhängig, dass sich der Sachverhalt im Anwendungsbereich des Unionsrechts abspielt; sie ist also mit der Regelungszuständigkeit der Union verknüpft, die gerade bei sozialen Rechten nur punktuell wahrgenommen wird ${ }^{28}$ oder nur eingeschränkt besteht. Der EuGH kann dann den Schutz sozialer Rechte in der

22 EuGH, Gutachten 2/13 vom 18.12.2014, Rn. 164; vgl. dazu: V. Skouris, Die Europäische Grundrechtcharta in der Rechtsprechung des EuGH, AuR 2015, S. 294, 297; C. Grabenwarter, Das EMRK-Gutachten des EuGH, EuZW 2015, S. 180; C. Tomuschat, Der Streit um die Auslegungshoheit, EuGRZ 2015, S. 133; M. Breuer, Wasch mir den Pelz aber mach mich nicht nass, EuR 2015, S. 330.

23 EuGH, Urteil v. 26.2.2013, Rs. C-399/11 (Melloni), ECLI:EU:C:2013:107, Rn. 60.

24 Kritisch: M. Breuer (Fn. 22), S. 330, 334.

25 C. Ladenburger, in: Tettinger/Stern (Fn. 3), Art. 51 GRC, Rn. 58; dazu auch die Entschließung des Europäischen Parlaments zum Bericht über die Situation der Grundrechte in der EU, Rn. 13: (The EP) „,calls on the Commission to give more priority to the preparation of the Union's accession to the European Social Charter...".

26 S. Krebber, Die Bedeutung der Grundrechtecharta und der EMRK für das deutsche Individualarbeitsrecht, EuZA 2013, S. 188, $190 \mathrm{f}$.

27 EuGH, Urteil v. 15.1.2014, Rs. C-176/12 (AMS), ECLI:EU:C:2014:2, Rn. 46-48.

28 EuGH, Urteil v. 16.1.2008, Rs. C-361/07 (Polier), ECLI:EU:C:2008:16, Slg. 2008, I-00006; Urteil v. 5.2.2015, Rs. C-117/14 (Nistahuz Poclava), ECLI:EU:C:2015:60. 
EU nur unvollständig gewährleisten; ${ }^{29}$ umgekehrt soll die Wirkung des Unionsrechts abweichende Grundrechtsgewährleistungen in den Mitgliedstaaten effektiv einschränken.

\section{Einfluss des Arbeitsvölkerrechts auf soziale Grundrechte der GRC}

Beim Schutz sozialer Grundrechte ist das Konfliktpotential zwischen Unionsrecht und dem Völkervertragsrecht größer als bei bürgerlichen und politischen Rechten. Dies ergibt sich aus der unklaren Stellung sozialer Rechte in der GRC ${ }^{30}$, die von vielen als bloße „Grundsätze“ verstanden werden ${ }^{31}$, auch wenn dies keinesfalls für alle Rechte dieses Titels unterstellt werden darf. In der ESC gelten ausdifferenzierte Grundrechtsgewährleistungen, wenn auch in Form einer Staatenverpflichtung. Festzuhalten bleibt allerdings, dass auch auf Ebene des Europarats soziale Rechte nicht ebenso wie die bürgerlichen und politischen Rechte geschützt werden, was bereits die Aufteilung der Gewährleistung von Menschenrechten auf zwei getrennte Instrumente (EMRK und ESC) mit unterschiedlichen Ratifizierungsbedingungen und unterschiedlichen Überwachungsmechanismen belegt.

Auf der anderen Seite bekennen sich die Mitgliedstaaten des Europarats zur „Unteilbarkeit" von Menschenrechten, die auf der Anerkennung ihrer notwendigen Wechselbezüglichkeit beruht ${ }^{32}$ : Ohne soziale Grundrechte gelangen auch die bürgerlichen und politischen Rechte nicht zur Entfaltung. Infolge dieser Wechselbezüglichkeit hat sich die Rechtsprechung des EGMR zu einer kooperationsfreundlichen Angleichung internationaler Auslegungsstandards bekannt. ${ }^{33}$ Für die sozialen Rechte war die bedeutendste Folge dieser Methode die Weiterentwicklung des Grundrechts der Vereinigungsfreiheit in Art. 11 EMRK $^{34}$ zu einem sozialen Recht auf Tarifverhandlungen ${ }^{35}$ und Arbeitskampf ${ }^{36}$. Dass diese Rechte bereits in Art. 6 ESC verankert sind, technisch also gerade dem komplementären Regelungsinstrument zugewiesen wurden, hat diese Entwicklung zwar zeitlich verzögern, aber nicht aufhalten können.

$\mathrm{Zu}$ einer Übernahme von Inhalt und Schranken der Garantien der ESC in die EMRK führt diese Interpretation jedoch keineswegs. Der EGMR selbst hat keinen zwingend einheitlichen europäischen Schutzstandard für soziale Grundrechte

29 S. Krebber (Fn. 26), S. 188, 199.

30 C. Ladenburger, in: Tettinger/Stern (Fn. 3), Art. 52 GRC, Rn. 7 f.

31 Kritisch: M. Delfino, The Court and The Charter, European Labour Law Journal 2015, S. 86, 89 ff.

32 M. Schlachter (Fn. 14), S. 105, $115 \mathrm{ff}$.

33 EGMR, Urteil v. 4.6.2015 - 51637/12 (Chitos/Greece), RJD 2015, Rn. 45-52, interpretiert das Verbot der Zwangsarbeit in Art. 4 EMRK nach Auseinandersetzung mit dem Gewährleistungsgehalt anderer Normen des Arbeitsvölkerrechts.

34 A. Nußberger, Die Rechtsprechung des EGMR zur Koalitionsfreiheit, DRdA 2015, S. 408, 409; zum Einfluss der ILO vgl. J. Heuschmid, SR 2014, S. 1 ff.; M. Schlachter in: Däubler/Zimmer (Hrsg.), Festschrift für K. Lörcher, 2013, S. 302, $303 \mathrm{ff}$.

35 EGMR (Große Kammer), Urteil v. 12.11 .2008 - 34503/97 (Demir und Baykara/Turkey), RJD 2008-V, Rn. 147.

36 EGMR, Urteil v. 8.4.2014 - 31045/10 (National Union of Rail Maritime and Transport Workers (RTM)/UK), RJD 2014-II, Rn. 84; Urteil v. 2.10.2014 - 48408/12 (Tymoshenko/Ukraine), Rn. 78. 
schaffen wollen ${ }^{37}$, sondern das Arbeitsvölkerrecht lediglich als Instrument zur Auslegung einer verwandten Gewährleistung in der EMRK herangezogen. Die Besonderheiten der Konvention, zu denen meistens ein weiter Beurteilungsspielraum der Konventionsstaaten $z_{a ̈ h l t}{ }^{38}$, ziehen internationalen Vereinheitlichungsbemühungen Grenzen. ${ }^{39}$ Selbst wenn sich im Arbeitsvölkerrecht bereits eine langjährige, einheitliche Auslegungspraxis entwickelt hat ${ }^{40}$ (im Anlassfall: die Anerkennung von Unterstützungsstreiks als Bestandteil der Arbeitskampffreiheit), wird diese nicht zwingend übernommen; der EGMR hält bei der Interpretation von Art. 11 EMRK an einem weiten Ermessensspielraum der Konventionsstaaten fest $^{41}$, der ein Verbot von Unterstützungsstreiks im besonderen Fall rechtfertigen kann.

Dennoch hat die Methode des EGMR einen Überschneidungsbereich zwischen bürgerlichen und sozialen Grundrechten geschaffen, in dem soziale Rechte aus der ESC an der Garantie des Unionsrechts für den Schutzstandard der EMRK teilhaben. Soweit der EGMR den Inhalt sozialer Rechte unter Berücksichtigung ihrer Quellen in der ESC bestimmt, schlägt die Angleichung der Auslegungsstandards insoweit auf die GRC durch. ${ }^{42}$ Ohne einen Beitritt der Union zur EMRK kann diese jedoch nicht selbständig Grundrechte für EU-Bürger schaffen, die sich für die Wahrnehmung eines Streikrechts gegenüber der Union folglich nicht unmittelbar auf Art. 11 EMRK berufen können. Rechtsgrundlage bleibt im Anwendungsbereich des Unionsrechts allein Art. 28 GRC. Nur soweit der EGMR die Bestimmungen der ESC seiner Auslegung der EMRK zugrunde legt ${ }^{43}$, kann diese indirekt auch die Interpretation der GRC beeinflussen.

Dieser ohnehin begrenzte mittelbare Einfluss der ESC relativiert sich weiter dadurch, dass der Schutzbereich von Art. 28 GRC im Unionsrecht begrenzt wird. ${ }^{44}$ An die Unionsorgane als Grundrechtsadressaten, denen die Kompetenz zur Regelung des Koalitions- und Arbeitskampfrechts fehlt (Art. 153 Abs. 5 AEUV) richtet sich Art. 28 GRC vornehmlich dann, wenn es um die Einschränkung dieser Rechte durch Sekundärrecht geht. ${ }^{45}$ Mithin erfolgen Konkretisierung und Ausgestaltung des Tarif- und Arbeitskampfrechts im Wesentlichen auf der Ebene der Mit-

37 EGMR, Urteil v. 8.4.2014-31045/10 (Fn. 36), Rn. 92-98.

38 EGMR, Urteil v. 8.4.2014 - 31045/10 (Fn. 36), Rn. 86; dazu: T. v. Danwitz, in: Tettinger/Stern (Fn. 3), Art. 52 GRC, Rn. 24.

39 A. Nußberger (Fn. 34), S. 408, 410.

40 Darauf stellt entscheidend ab: M. Breuer, in: Grabenwarter (Hrsg.), Europäischer Grundrechteschutz, Enzyklopädie Europarecht, Bd. 2, 2014, § 7, Fundamentalgarantien, Rn. 61.

41 EGMR, Urteil v. 8.4.2014 - 31045/10 (Fn. 36), Rn. 86, 99.

42 T. v. Danwitz, in: Tettinger/Stern (Fn. 3), Art. 52 GRC, Rn. 57.

43 EGMR (Große Kammer), Urteil v. 12.11.2008 - 34503/97 (Demir and Baykara/Turkey), RJD 2008-V, Rn. 85: "the consensus emerging from specialized international instruments ... may constitute a relevant consideration for the Court when interpreting the provisions of the Convention in specific cases"; mit Verweis auf die ESC auch EGMR, Urteil v. 2.10.2014 - 10609/10 (Matelly/France), Rn. 32, 33.

44 A. Seifert, Die Bedeutung von EMRK und GRC für das deutsche kollektive Arbeitsrecht, EuZA 2013, S. 205, 211.

45 R. Patett, Das Verhältnis des Arbeitskampfrechts zu Art. 12 GG und den europäischen Grundfreiheiten, 2015, S. 190 f., 200. 
gliedstaaten; dies allerdings nur, soweit das Unionsrecht dem nicht entgegensteht. Von Vorgaben des Unionsrechts sind sie dabei nicht entbunden ${ }^{46}$, denn die Kompetenzschranke des Art. 153 Abs. 5 AEUV kann Anforderungen des Primärrechts einschließlich der GRC nicht beseitigen; dasselbe gilt für einzelstaatliche Maßnahmen, die durch ihren Grundfreiheitenbezug in den Anwendungsbereich des Unionsrechts fallen.

Insgesamt ist der Einfluss, den die ESC im Unionsrecht auf eine effektive Gewährleistung sozialer Rechte gewinnen könnte, schwach ausgeprägt. Eine Auslegung von Grundrechten als Mindestgewährleistung bleibt möglich, ist aber nur für die EMRK-Rechte vorgeschrieben, die diesen Status den sozialen Rechten nur sehr begrenzt vermitteln. Die in Art. 53 GRC in Bezug genommenen völkerrechtlichen Abkommen werden weder Bestandteile der GRC noch Mindeststandard für deren Inhalte. Dies kann durchaus mit der Überlegung hinterfragt werden, warum die Norm als Anwendungsvoraussetzung einen Beitritt aller EU-Mitgliedstaaten fordert, der praktisch einen bereits allseits akzeptierten Mindeststandard garantiert, wenn die Union daraus für die Gewährleistungen der GRC keine Konsequenzen ziehen möchte. Methodisch scheint es aber kaum vertretbar, den sprachlichen Unterschied in den Gewährleistungen des Schutzniveaus in Art. 52 Abs. 3 und Art. 53 GRC außer Betracht zu lassen; in zeitlicher Nähe zum Inkrafttreten der GRC wird davon auszugehen sein, dass die GRC eine umfassendere Garantie für Rechte aus der EMRK als für andere völkerrechtliche Verträge begründet. Für die Union werden soziale Rechte der ESC lediglich für die Auslegung inhaltlich ihr entsprechender Grundrechte berücksichtigt. ${ }^{47}$

Bei Maßnahmen der Mitgliedstaaten gilt dasselbe, soweit sie sich im Anwendungsbereich des Unionsrechts bewegen, der GRC also zumindest teilweise unterliegen. Nur mitgliedstaatliche Maßnahmen, die außerhalb des Anwendungsbereichs des Unionsrechts bleiben, unterliegen ausschließlich den Vorgaben der Verfassung und des Arbeitsvölkerrechts. Bestehenden völkerrechtlichen Verpflichtungen gegenüber kann sich ein Staat nicht wirksam auf den Vorrang des Unionsrechts berufen. ${ }^{48}$ Zwar ist die Union selbst an die ESC nicht gebunden, muss also ihre Rechtsakte oder Maßnahmen an deren Gewährleistungen nicht ausrichten; auf die Gebundenheit der Mitgliedstaaten strahlt das aber nicht aus. In vergleichbarer Form hat auch der EGMR unterstrichen, dass die Mitgliedschaft in einer internationalen Organisation wie der EU die Mitgliedstaaten von den Verpflichtungen aus der EMRK nicht entbinden kann. ${ }^{49}$ Stattdessen hat der EGMR jedoch eine umfassende Vermutung der Vereinbarkeit von Unionsrecht mit der

46 Anders: A. Sagan, Gemeinschaftsgrundrecht auf Kollektivmaßnamen, 2008, S. $129 \mathrm{ff}$.

$47 \mathrm{~J}$. Kokott/C. Sobotta, Die Charta der Grundrechte der Europäischen Union nach dem Inkrafttreten des Vertrags von Lissabon, EuGRZ 2010, S. 265, 267; kritisch: S. Krebber, Soziale Rechte in der Gemeinschaftsrechtsordnung, RdA 2009, S. 224, 235; R. Rebhahn in: Grabenwarter (Hrsg.), Europäischer Grundrechteschutz, 2014, § 16, Rechte des Arbeitslebens, Rn. 42.

48 EGMR (Große Kammer), Urteil v. 18.2.1999 - 24833/94 (Matthews/UK), RJD 1999-I, Rn. 32 f.

49 EGMR (Große Kammer), Urteil v. 30.6.2005 - 45036/98 (Bosphorus/Ireland), RJD 2005-VI. 
EMRK aufgestellt. Entsprechend können Mitgliedstaaten sich durch Verweis auf die Vereinbarkeit grundrechtsrelevanter Maßnahmen mit dem Unionsrecht gegen eine Kontrolle an der EMRK schützen. ${ }^{50}$ In das Verhältnis des Unionsrechts zur ESC ist diese Lösung jedoch nicht übertragbar. ${ }^{51}$ Solange soziale Rechte der ESC nicht als Mindeststandard für die Auslegung von Unionsgrundrechten anerkannt werden, also eine Annäherung des Gewährleistungsumfangs von Art. 52 Abs. 3 und Art. 53 GRC nicht stattfindet, fehlt eine tatsächliche Grundlage für eine vergleichbare umfassende Vereinbarkeitsvermutung.

\section{Möglichkeiten zur Verstärkung des Schutzes sozialer Rechte in der Union}

Das Ziel der GRC, auch für soziale Rechte einen kollisionsfreien Schutz im Mehr-Ebenen-System zu garantieren, ist unter den gegenwärtigen Bedingungen gefährdet. Die Besonderheiten der Union als einer supranationalen Institution stehen einer Übernahme der materiellen Angleichung von Grundrechtsgewährleistungen vielfach entgegen. Davon wird eine Übertragung der methodischen Ansätze des EGMR in die Rechtsprechung der Union nicht ausgeschlossen. Für eine Verstärkung des Schutzes sozialer Rechte kommen dafür insbesondere zwei Ansatzpunkte in Betracht: Eine systematische Berücksichtigung des Arbeitsvölkerrechts bei der Interpretation sozialer Grundrechte ${ }^{52}$, und die Übernahme der Schutzpflichtenlehre für ihre Auslegung. Beides ist dem EuGH nicht fremd, wird bislang aber für die Entwicklung des Schutzstandards sozialer Rechte nicht umfassend genutzt.

Zwar hat der EuGH vor Inkrafttreten der GRC seine Grundrechtsrechtsprechung auch unter Berücksichtigung völkervertragsrechtlicher sozialer Rechte entwickelt $^{53}$, seitdem ist eine „Inspirationsquelle“ für die Anerkennung von sozialen Grundrechten in der Union aber nicht mehr erforderlich. Entsprechend werden solche Rechtsquellen nur selten herangezogen, und wenn dies der Fall ist ${ }^{54}$, überwiegend in Form eines Zitats zur Bestätigung einer bereits aus dem Unionsrecht abgeleiteten Interpretation. ${ }^{55}$ Zur Gewinnung eines Auslegungsergebnisses wird das Arbeitsvölkerrecht kaum verwendet; es nimmt entsprechend keinen Einfluss auf den Inhalt. ${ }^{56}$ Noch weniger würde eine Entscheidung zur Abweichung von

50 EGMR (Große Kammer), Urteil v. 30.6.2005 - 45036/98 (Fn. 49), Rn. 147, 155.

51 European Committee of Social Rights, Entscheidung im Kollektivbeschwerdeverfahren Nr. 55/2009 (CGT/ France), Rn. 35.

52 EGMR, Urteil v. 2.10.2014 - 10609/10 (Fn. 43), Rn. 32, 33; EGMR, Urteil v. 8.4.2014 - 31045/10 (Fn. 36), Rn. 96, 97.

53 EuGH, Urteil v. 15.6.1987, Rs. C-149/77 (Defrenne III), ECLI:EU:C:1978:130, Slg. 1978, 01365; Urteil v. 11.12.2007, Rs. C-438/05 (Viking Line), ECLI:EU:C:2007:772, Slg. 2007, I-10779, Rn. 43; Urteil v. 18.12.2007, Rs. C-341/05 (Fn. 17), Rn. 90.

54 EuGH, Urteil v. 15.4.2008, Rs. C-268/06 (Impact), ECLI:EU:C:2008:223, Slg. 2008, I-02483, Rn. 113; EuGH, Urteil v. 15.7.2010, Rs. C-271/08 (Fn. 9), Rn. 37.

55 V. Skouris (Fn. 22), S. 294, 295.

56 J. Heuschmid (Fn. 34), S. 1 ff. 
völkerrechtlichen Schutzstandards unter Erarbeitung der dafür maßgeblichen Besonderheiten des Unionsrechts begründet. Auf dieser Auslegungsstufe könnte eine Annäherung an die vom EGMR verfolgte Interpretationsmethode eine stärkere Konvergenz internationaler Standards ermöglichen. Den Besonderheiten des Unionsrechts kann dabei entsprochen werden, doch würden die Staaten nur in entsprechend begründeten Fällen dem Risiko kollidierender Grundrechtsanforderungen ausgesetzt.

Eine Übertragung der Schutzpflichtenlehre des EGMR (,positive obligations “57) in den Anwendungsbereich der GRC würde den Grundrechten neben ihrer Funktion der Abwehr staatlicher Eingriffe die positive Dimension beilegen, Bürger vor drohenden Rechtsverletzungen durch Dritte zu schützen. Obwohl diese Schutzpflicht grundsätzlich dem Gesetzgeber aufgegeben ist, gewährleistet die Rechtsprechung durch Auslegung und Rechtsfortbildung den erforderlichen Mindestschutz, wenn es an konkreten Schutznormen fehlt. Als Beispiel für die Anwendung auf soziale Rechte ist an die Gleichbehandlung grenzüberschreitend Beschäftigter mit den im Aufnahmemitgliedstaat geltenden Standards zu denken. Dies ist im Unionsrecht grundsätzlich über die Grundfreiheit der Arbeitnehmerfreizügigkeit vorgesehen, Art. 45 AEUV, wird aber in der Entsendesituation durch die Entsende-RL 96/71/EG ausgeschlossen. ${ }^{58}$ Diese Ausnahme zum Nachteil entsandter Arbeitnehmer wird im Arbeitsvölkerrecht überwiegend nicht geteilt. ${ }^{59}$ Auch Art. 19 ESC geht grundsätzlich von einer Pflicht zur Gleichbehandlung aller grenzüberschreitend tätigen Beschäftigten mit den inländischen Beschäftigten aus, erlaubt aber für bestimmte Rechte eine Differenzierung je nach der Aufenthaltsdauer am Arbeitsort. Würde hier den Unionsorganen bei der Begrenzung der Freizügigkeit die Beachtung von Schutzpflichten zugunsten des Grundrechts aufgegeben, könnten auch bei Schaffung des Sekundärrechts differenziertere Lösungen verlangt werden als eine vollständige Ausnahme entsandter Arbeitnehmer aus dem Gleichbehandlungsanspruch.

Eine neue Entwicklung bedeutet die Schutzpflichtenlehre für das Unionsrechts nicht. Zur Auslegung der Warenverkehrsfreiheit hat der EuGH diese Rechtsfigur bereits herangezogen ${ }^{60}$; einer Übertragung auf soziale Grundrechte stehen keine zwingenden Argumente entgegen. Auf die in Art. 51 Abs. 1 S. 2 GRC verankerte Verpflichtung der Union, die Anwendung der Grundrechte und Grundsätze ,zu fördern“ kann durchaus eine Pflicht zur Verwirklichung und Umsetzung aller

57 EGMR, Urteil v. 27.10.1994 - 18535/91 (Kroon/Netherlands), Serie A Nr. 297-C; EGMR (Große Kammer), Urteil v. 12.7.2002 - 28957/95 (Goodwin vs. UK), RJD 2002-VI.

58 Kritisch: H. Verschueren, The European internal market and the competition between workers, European Labour Law Journal 2015, S. 128, 135.

59 Vgl. ILO Migration for Employment Convention (Revised) No. 97, 1949; International Convention on the Protection of All Migrant Workers and their Families, 1990; näher zu den verschiedenen Standards der Gleichbehandlung im internationalen Recht: N. Countouris/S. Engblom: Protection or Protectionism?, European Labour Law Journal 2015, S. 20, 35 ff.; M. Schlachter: Kollektive Rechte bei Arbeitsmigration und Entsendung, in: Mulder (Hrsg.), Festschrift für Stein Evju, demnächst.

60 EuGH, Urteil v. 12.6.2003, Rs. C-112/00 (Schmidberger), ECLI:EU:C:2003:333, Slg. 2003, I-05659; dazu: $R$. Patett (Fn. 45), S. 240 f. 
GRC-Gewährleistungen gestützt werden. ${ }^{61}$ Schutzpflichten verletzen nicht die Bindung der GRC an die bestehenden Kompetenzgrenzen der Union, denn sie schaffen keine neuen Pflichten für die Mitgliedstaaten über das hinaus, was im Anwendungsbereich des Unionsrechts ohnehin geschuldet ist, nämlich die Gewährleistung des grundrechtlichen Mindeststandards. Insbesondere überlassen sie der EU oder den Mitgliedstaaten als Adressaten die Wahl geeigneter Schutzmechanismen und achten damit ihr gestalterisches Ermessen. Beide Ansätze können denkbare Kollisionen der Gewährleistung sozialer Rechte in Europa verringern und eine Entwicklung konvergenter Schutzstandards erleichtern. 